Ann. Zootech., 1986, 35 (4), 401-410

\title{
Sur l'intérêt de la mesure du transit digestif chez le lapin à l'aide de particules indigestibles
}

\author{
T. BOUYSSOU, M. CANDAU ${ }^{*}$ et Y. RUCKEBUSCH \\ Ecole Nationale Vétérinaire, Physiologie, 31076 Toulouse Cedex \\ * Ecole Nationale Supérieure Agronomique, 145, avenue de Muret, 31076 Toulouse Cedex
}

\begin{abstract}
Résumé
Le développement du segment caeco-colique ainsi que l'émission de deux types de fèces compliquent la mesure du flux digestif chez le lapin pour lequel l'emploi d'une méthode à double marquage semble indiqué.

En vue d'estimer les variations spontanées ou provoquées du transit digestif à court terme, le temps de transit moyen (T.T.M.) de particules de polyéthylène (P.P.E.) a été comparé à celui d'un colorant, la phénolsulphonephtaléine (P.S.P.) pour le segment gastro-iléal et le segment caeco-colique. Le double marquage a été effectué à des moments différents de la journée, de manière à différencier les périodes d'émission de crottes dures de celles correspondant à la production de caecotrophes.

Le T.T.M. des P.P.E. (phase solide) est supérieur d'environ 20 p. 100 par rapport à celui de la P.S.P. (phase liquide) pour le segment gastro-iléal. Les T.T.M. des P.P.E. et de la P.S.P. sont très voisins pour le segment caeco-colique dans le cas de l'émission de caecotrophes. Le T.T.M. des P.P.E. est inférieur d'environ 20 p. 100 à celui de la P.S.P. dans le cas des crottes dures.

La mesure du T.T.M. à l'aide des P.P.E. voisin de $90 \mathrm{mn}$ s'avère satisfaisante pour apprécier le flux digestif du segment gastro-iléal quelle que soit la période du nycthémère. Un T.T.M. de P.P.E. inférieur à $2 \mathrm{~h}$ au lieu de $4 \mathrm{~h}$ pour le segment caeco-colique implique l'émission de fèces molles et s'observe durant la phase diurne du nycthémère. L'intérêt de cette méthode de marquage de la phase solide par des P.P.E. est sa validité pour l'identification de la dualité de l'excrétion fécale.
\end{abstract}

Mots clés : Transit, particules indigestibles, caecotrophie.

\section{Introduction}

L'analyse du transit digestif global chez le lapin est relativement complexe en raison du développement anatomofonctionnel du segment cæco-colique (LAPLACE et al., 1974, 1975).

Le cæcum joue en effet le rôle d'un réservoir où le contenu iléal pourra séjourner pendant plusieurs heures, le temps de rétention apparent d'un marqueur de la phase solide comme le $\mathrm{Cr}_{2} \mathrm{O}_{3}$ étant voisin de 6 heures chez le lapin en croissance (GIDENNE \& PONCET, 1985). 
Quant au côlon, il est responsable de la dualité de l'excrétion fécale dans laquelle les phases solide et liquide sont dissociées lors de la formation des crottes dures (Pickard \& Stevens, 1972).

L'interférence de multiples facteurs, tels que le rythme spontané des prises alimentaires (PruD'hon et al., 1972), la finesse de broyage des constituants d'un aliment granulé (LAPLACE \& LEBAS, 1977), amènent la plupart des auteurs à considérer comme indispensable l'emploi d'au moins deux marqueurs, l'un pour la phase solide, l'autre pour la phase liquide.

De surcroît, il peut être intéressant d'envisager séparément les temps de transit pour les segments antérieurs et postérieurs à la jonction iléo-cæacale lorsqu'on examine l'influence de facteurs modifiant l'évacuation gastrique ou l'activité cæco-colique.

Le nombre restreint d'études de transit réalisé chez le lapin nécessite la pratique des abattages et fait appel à des techniques généralement très lourdes, telles que l'utilisation de cérium (LAPLACE et al., 1974) ou la technique de mordançage du chrome (UDEN et al., 1980). Chez l'homme, la mesure du temps de transit de particules indigestibles s'est avérée des plus satisfaisantes, et cela quelle que soit la densité des particules pour identifier l'évacuation gastrique et le transfert jéjuno-iléal (BECHGAARD et al., 1985) ou pour mesurer les temps de transit global et détecter les constipations idiopathiques (Lawson, 1986 ; Chaussade et al., 1986). Chez le chien et le porc, l'incorporation de particules indigestibles à une concentration inférieure à $10 \mathrm{p}$. $100 \mathrm{de}$ la matière sèche ne modifie pas la motricité digestive et permet une mesure du temps de transit, y compris une vitesse accrue du transit colique lors de la diminution de l'activité antipéristaltique du côlon (Cherbut \& Ruckebusch, 1984, 1985).

Nous avons donc vérifié, chez le lapin, l'intérêt de l'utilisation de particules indigestibles pour la mesure du transit gastro-iléal d'une part et cæco-colique d'autre part, le but de l'expérimentation étant de connaître le degré de fiabilité d'un tel procédé pour apprécier par exemple l'influence de la granulométrie sur le transit gastro-intestinal (Pairet et al., 1986a) ou encore les modifications du temps de transit au cours de la production de crecotrophes (PAIRET et al., 1986b).

L'étude a été réalisée par comparaison à la progression, dans le tube digestif, d'un marqueur de la phase liquide, la phénolsulfonephtaléine (P.S.P.). Les marqueurs ont été administrés par voie orale ou iléale, à des moments différents de la journée, de manière à y inclure les périodes naturelles de cæcotrophie.

\section{Matériel et méthodes}

Huit lapins adultes (femelles âgées de 1 an et pesant de 3 à $4 \mathrm{~kg}$ ), de race néozélandaise, et élevés en cage individuelle, ont été utilisés sur une période de 4 mois. Ils reçoivent, ad libitum, un aliment granulé du commerce $(14$ p. 100 de protéines et 16 p. 100 de cellulose brute). Chacun des sujets est muni, sous anesthésie générale après une période de jeûne de $24 \mathrm{~h}$, d'une canule gastrique ou iléale en pyrex (4 lapins pour chaque canule). La canule gastrique droite $(6 \mathrm{~mm}$ de diamètre intérieur, $2 \mathrm{~mm}$ d'épaisseur et $6 \mathrm{~cm}$ de longueur) est maintenue à l'intérieur de la paroi fundique grâce à un rebord de $2 \mathrm{~mm}$, le long de la grande courbure, $3 \mathrm{~cm}$ en aval du cardia. La canule 
iléale en $\mathrm{T}$ ( $4 \mathrm{~mm}$ de diamètre intérieur, $2 \mathrm{~mm}$ d'épaisseur) est maintenue dans la lumière de l'iléon terminal, $5 \mathrm{~cm}$ en amont de la valvule iléo-cæcale par des rebords semi-cylindriques $(10 \mathrm{~mm})$. Les mesures de l'évacuation gastrique, du transit boucheiléon et iléon-fèces ont été effectuées dès le retour à une consommation alimentaire jugée normale (15 à 20 jours selon les individus) par pesée régulière des animaux, la consommation d'aliment, d'eau et le poids des fèces.

Les lapins pris dans leur cage d'élevage, sans jeûne préalable, sont maintenus dans des cages à contention, à fond grillagé, se refermant par un système de guillotine, empêchant toute possibilité de cæcotrophie et sans accès à la nourriture.

Deux marqueurs ont été utilisés : la phénolsulfonephtaléine (P.S.P.) $(1 \mathrm{ml}$ de la solution, $25 \mathrm{mg} / \mathrm{ml}$ ) et des particules de polyéthylène sphériques (P.P.E.) de densité égale à 1,06 et de diamètre voisin de $1 \mathrm{~mm}$, permettant ainsi l'usage d'une sonde de silastic de $3 \mathrm{~mm}$ de diamètre intérieur, $1 \mathrm{~mm}$ d'épaisseur et $10 \mathrm{~cm}$ de longueur.

Vingt particules de P.P.E. (selon Pickard \& Stevens, 1972) ont été administrées par sonde œsophagienne pour l'étude du transit bouche-iléon et directement dans la canule iléale pour l'étude du transit iléon-fèces.

L'injection des deux marqueurs est faite simultanément. Cette dernière n'est pas nécessairement liée à un repas, le lapin étant en état de réplétion quasi permanente dans des conditions d'alimentation ad libitum.

Lors de la vidange gastrique, cinq minutes après l'administration de P.S.P., un premier prélèvement de contenu gastrique est effectué, et sa concentration est considérée maximale au temps zéro. Des échantillons de contenu gastrique $(3 \mathrm{ml})$ sont ensuite recueillis par gravité toutes les 30 minutes pendant 3 heures, puis 4 et 5 heures plus tard.

Les prélèvements de contenu iléal, pour le transit bouche-iléon, ont été obtenus par gravité et effectués en continu pendant 4 heures. Les tubes sont permutés toutes les 15 minutes, les volumes obtenus sont de $3 \mathrm{ml}$ en moyenne. De même, les prélèvements des fèces, pour le transit iléon-fèces, sont effectués en continu pendant 7 heures, grâce à des tubes placés sur un plateau rotatif, sous un entonnoir sur lequel repose la cage à contention. La libération contrôlée du plateau permet la collecte 30 minutes par 30 minutes des émissions fécales.

La concentration de P.S.P. est évaluée, dans la phase liquide des échantillons, par colorimétrie $(523 \mathrm{~mm})$ après centrifugation, dilution, défécation et alcalinisation par $\mathrm{Na}_{2} \mathrm{PO}_{4}$ des échantillons (BARREIRo et al., 1968).

Les résultats sont exprimés en pourcentage de la dose initiale injectée : Quantité de P.S.P. présente dans la phase liquide de l'échantillon $(\mathrm{mg}) \times 100$

Quantité de P.S.P. injectée, soit $25 \mathrm{mg}$

Les particules sont comptées dans le surnageant après dilution, défécation et centrifugation des échantillons prélevés. Le nombre de particules présentes dans chaque tube est rapporté aux vingt particules injectées.

Le temps de transit moyen (T.T.M.) est défini comme celui séparant le moment de l'administration du marqueur et le pic de sa concentration maximale (BARREIRo et al., 1968). 
Les transits ont été mesurés le matin à partir de $8 \mathrm{~h}$, l'après-midi à partir de $14 \mathrm{~h}$, et le soir à partir de $20 \mathrm{~h}$. Deux essais ont été effectués chez chacun des individus pour l'étude de la vidange gastrique et du transit bouche-iléon. Un essai sur chaque lapin a été effectué à partir de $14 \mathrm{~h}$ pour l'étude du transit iléon-fèces.

Un exemple de transit iléon-fèces, pendant la production de cæcotrophes, a été obtenu à partir de $8 \mathrm{~h}$.

Une mesure du temps de transit iléon-fèces a été réalisée à partir de $14 \mathrm{~h}$ sur trois lapins, pendant l'émission de cæcotrophes induite par une perfusion intraveineuse de prostaglandines $F_{2 \alpha}(10 \mu \mathrm{g} / \mathrm{kg} / \mathrm{mn}$ pendant $30 \mathrm{mn})$ selon un procédé antérieurement décrit (Pairet et al., 1986).

Les analyses statistiques ont été réalisées à partir du test non paramétrique de Mann et Whitney (SNEDECoR \& Cochran, 1967).

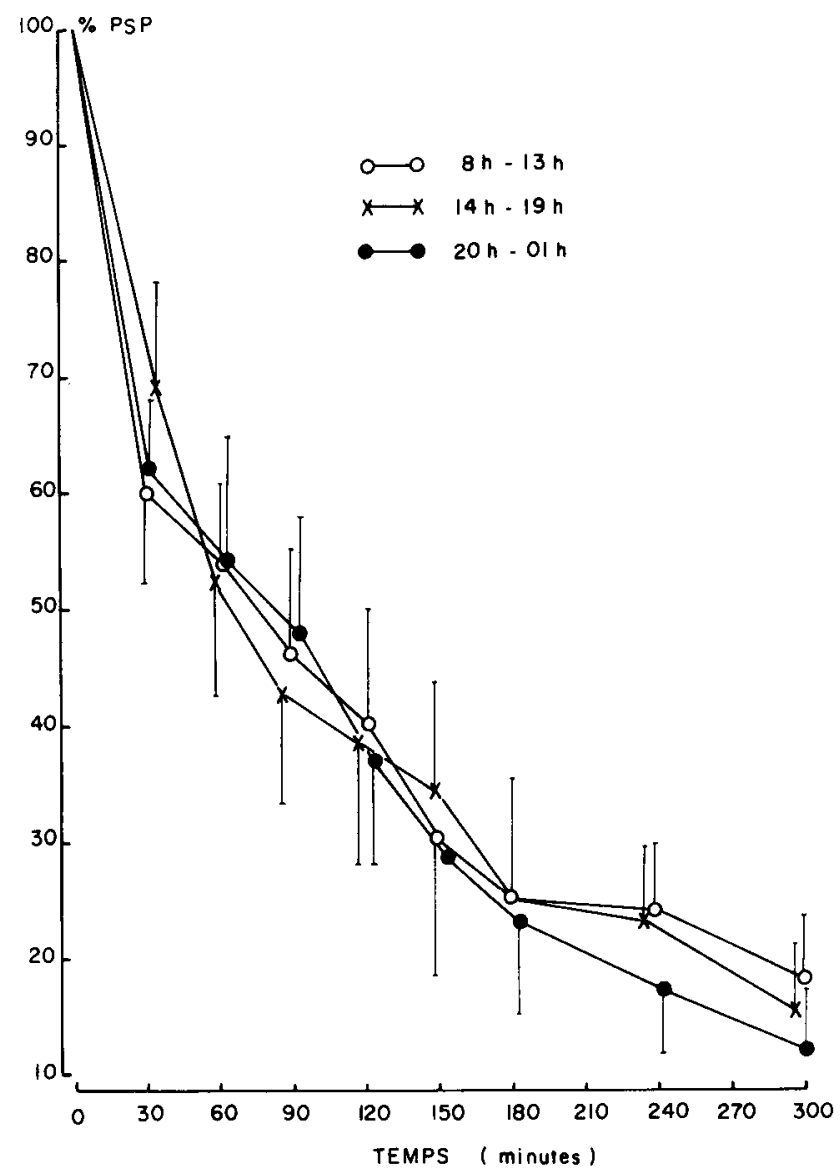

Fig. 1

Evolution de la concentration en P.S.P. dans des prélèvements de contenu gastrique effectués à $30 \mathrm{mn}$ d'intervalle après administration orale de P.S.P. à 8,14 et 20 heures.

Valeurs moyennes et écarts-types pour 8 essais effectués chez 4 lapins.

Decreases of P.S.P. concentration in the gastric contents collected at $30 \mathrm{mn}$ intervals after an oral administration at 8 a.m., 2 p.m. and 8 p.m. of a single dose of P.S.P. Mean and standard deviation for 8 trials in 4 rabbits. 


\section{Résultats}

\section{A. Evacuation gastrique de la phénolsulfone-phtaléine}

L'élimination de la P.S.P. administrée dans l'estomac est caractérisée par une décroissance de forme exponentielle (fig. 1), et ceci quelle que soit l'heure de l'administration.

Pendant les 30 premières minutes, environ 40 p. 100 de la P.S.P. sont évacués. Au cours d'une deuxième période, entre 30 et 180 minutes, l'élimination du marqueur est ralentie $(30$ p. 100) et devient négligeable $(10$ p. 100) durant les deux dernières heures.

\section{B. Transit bouche-iléon}

Le pourcentage de P.P.E. récupérées a été de $69 \pm 5$ p. 100 de la totalité des P.P.E. injectée. Il est nettement supérieur à celui de la P.S.P. qui est de $51 \pm 4$.

Le contenu iléal prélevé ne contient jamais plus de 10 à 12 p. 100 de la dose totale de P.S.P. administrée. Celle-ci apparaît 30 minutes après son administration. La quantité de P.S.P. du contenu iléal s'élève fortement 45 minutes après son administration œsophagienne à 8 heures et atteint son maximum $75 \pm 12$ minutes plus tard (fig. 2). Comme pour l'estomac, aucun effet de l'heure d'administration, sur le temps moyen du transit bouche-iléon, de la P.S.P. n'a été observé. Les valeurs maximales sont observées à $71 \pm 8$ minutes à $14 \mathrm{~h}$ et à $71 \pm 14$ minutes à $20 \mathrm{~h}$. Les valeurs individuelles sont extrêmement proches, avec un coefficient de variation inférieur à 7 p. 100 .

Les particules de polyéthylène (P.P.E.) suivent une courbe d'élimination iléale analogue à celle obtenue avec la P.S.P. (fig. 2), mais les variations individuelles sont plus importantes (coefficient de variation égal à 15 p. 100). Le contenu iléal prélevé ne contient jamais plus de 20 p. 100 de la quantité des particules de P.E. injectées. Cellesci semblent transiter de façon groupée, avec un maximum à $90 \pm 12$ minutes $(\mathrm{n}=8)$ après l'administration à $8 \mathrm{~h}, 96 \pm 14$ minutes à $14 \mathrm{~h}$ et $94 \pm 14$ minutes à $20 \mathrm{~h}$. Aucun effet de l'heure d'administration sur le temps de transit bouche-iléon n'est noté.

Comparées à la P.S.P., les particules de P.P.E. atteignent plus tardivement l'iléon $(90$ vs. $75 \mathrm{mn})(\mathrm{P}<0,05)$.

\section{Transit iléon-fèces}

Dans les échantillons, prélevés toutes les 30 minutes, la quantité de marqueur (mg de P.S.P. ou nombre de particules) n'excède pas (en moyenne) 12 à 15 p. 100 de la dose injectée de chacun d'eux.

La P.S.P. et les P.P.E. suivent une même courbe d'élimination, avec un décalage de 70 minutes $(P<0,05)$ (fig. 3).

Les mesures du transit iléon-fèces durant la nuit $(20 \mathrm{~h})$ sont du même ordre de grandeur $(\mathrm{P}<0,05)$. En revanche, la mesure du transit iléon-fèces réalisé à partir de $8 \mathrm{~h}$ chez un lapin qui n'a éliminé que des cæcotrophes, a fourni un pic de concentration 

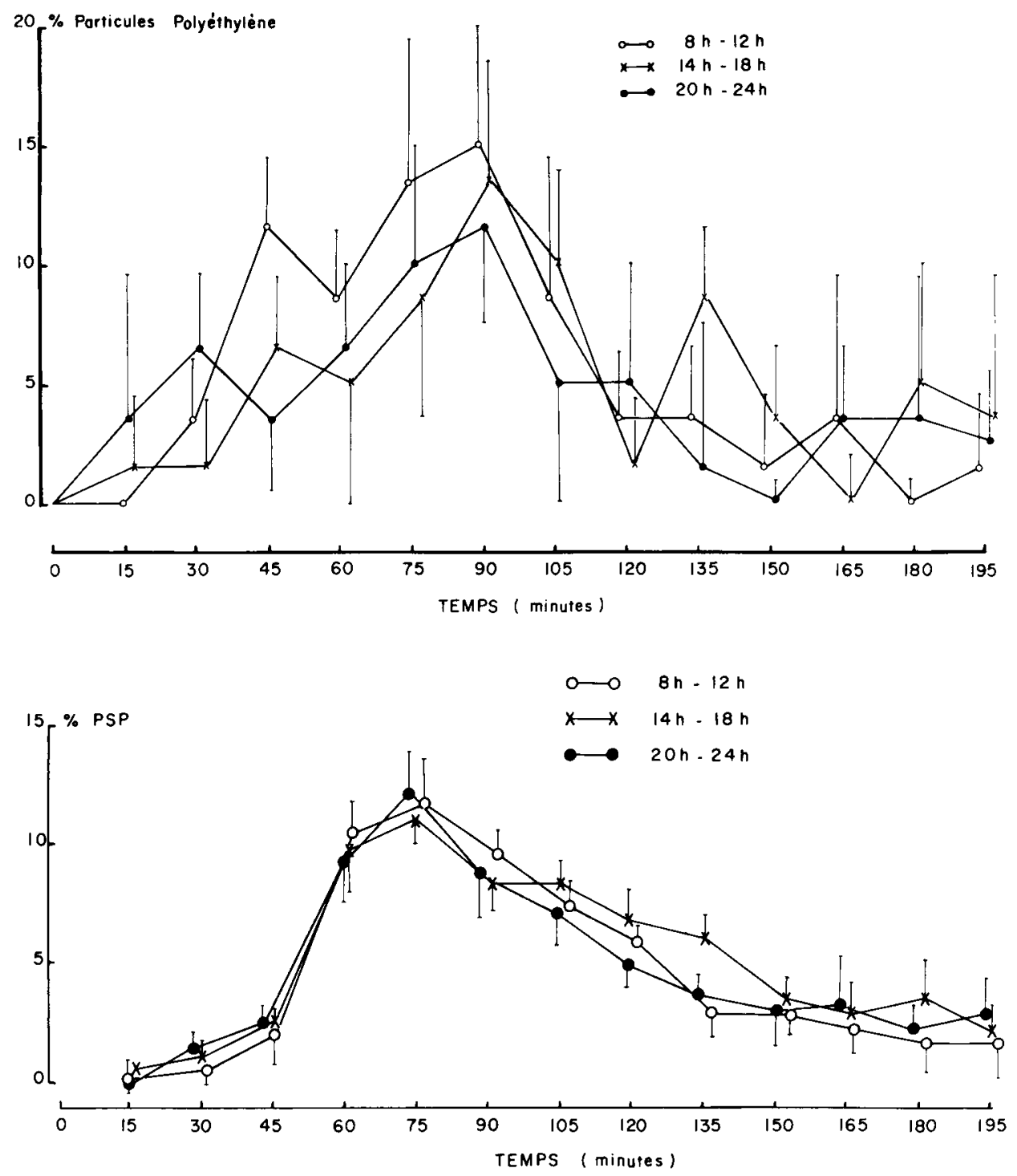

FIG. 2

Evolution de la quantité en polyéthylène et en P.S.P. du contenu iléal. Prélèvements effectués de 15 en $15 \mathrm{mn}$ après administration orale à 8,14 et 20 heures. Valeur moyenne et écart-type pour 8 essais chez 4 lapins.

Time course of P.S.P. and polyethylene (P.P.E.) quantities of ileal contents, sampled at $15 \mathrm{mn}$ intervals after a single oral dose of P.S.P. and P.P.E. at 8 a.m., 2 p.m. and 8 p.m. Mean and standard deviation for 8 trials in 4 rabbits. 


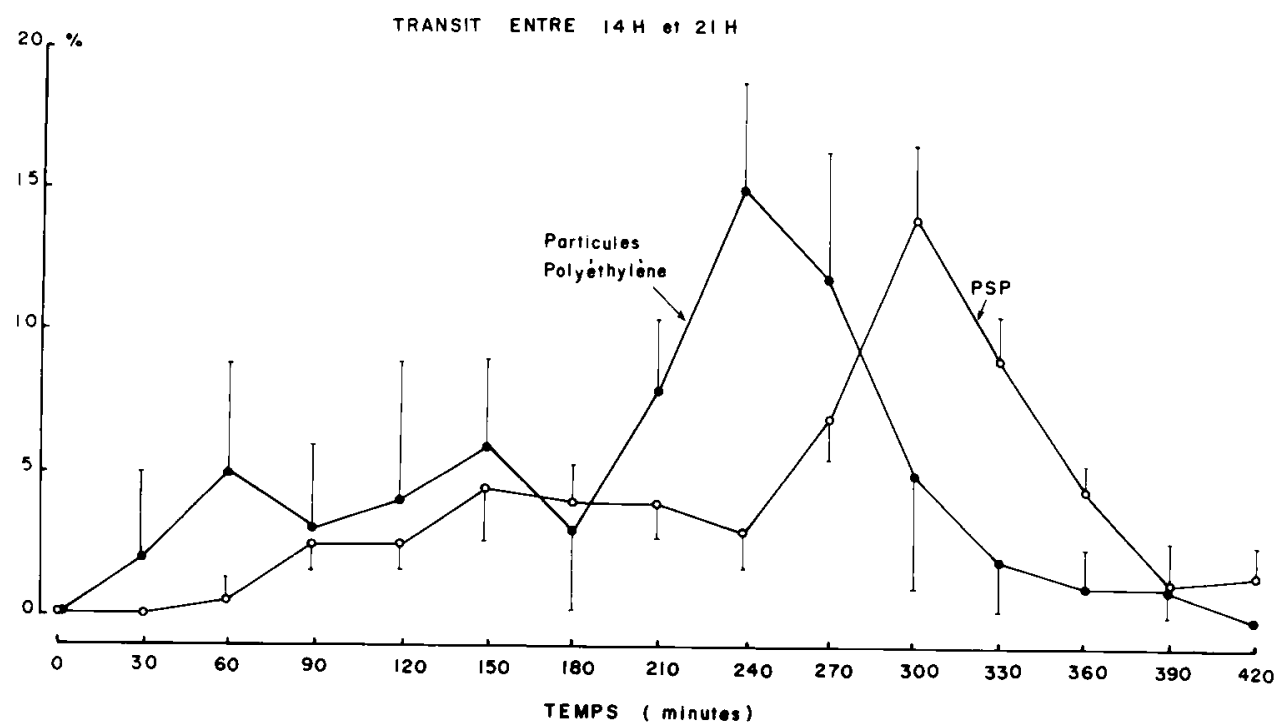

FIG. 3

Evolution de la quantité en polyéthylène et en P.S.P. lors d'émission de fèces dures. Prélèvements effectués de 30 en $30 \mathrm{mn}$ après administration iléale à 14 heures. Valeur moyenne et écart-type pour 4 essais chez 4 lapins.

Time course of P.S.P. and P.P.E. quantities during hard faeces production.

Sampling was performed at $30 \mathrm{mn}$ intervals after an ileal administration of the markers at 2 p.m. Mean and standard deviation for 4 trials in 4 rabbits.

pour la P.S.P. et les P.P.E. respectivement 90 et 120 minutes après leur administration (fig. 4). La quantité de P.P.E. atteint alors 25 p. 100 au lieu des 15 p. 100 dans le cas de l'émission des crottes dures.

Des temps de transit de P.P.E. voisins de 120 minutes : $112 \mathrm{mn}, 123 \mathrm{mn}, 145 \mathrm{mn}$, ont également été observés chez des lapins produisant des cæcotrophes à partir de $14 \mathrm{~h}$, à la suite de la perfusion intraveineuse durant 30 minutes de prostaglandines $F_{2 \alpha}$.

\section{Discussion et conclusion}

Le temps de transit moyen (T.T.M.) des P.P.E. s'avère supérieur à celui d'un marqueur de la phase liquide pour le segment digestif antérieur. Il convient cependant de souligner que le pourcentage total de P.P.E. récupérées $(69 \pm 5$ p. 100) est supérieur à celui de la P.S.P. (51 \pm 4 p. 100), pour laquelle on estime à près de 50 p. 100 la quantité fixée par adhérence à la muqueuse gastro-intestinale. En revanche, les valeurs sont moins homogènes pour les P.P.E. que pour les P.S.P. Cependant, il existe toujours un moment bien individualisé de plus grande élimination des particules qui est 


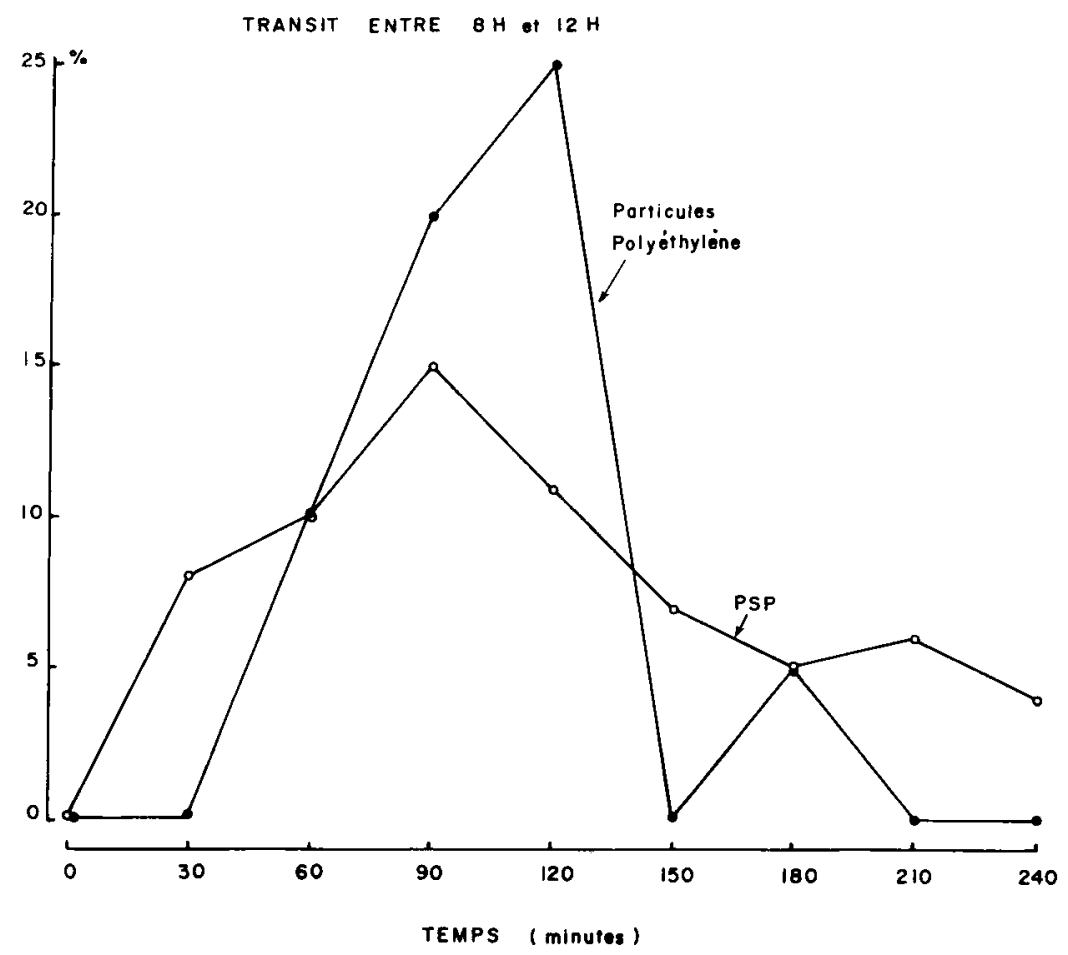

FIG. 4

Exemple d'évolution de la quantité en polyéthylène et en P.S.P. lors d'émission de fèces molles chez le lapin. Noter que la quantité est maximale pour les deux marqueurs de 90 à $120 \mathrm{mn}$ après leur administration.

Example of P.S.P. and P.P.E. elimination during soft faeces production in the rabbit. Note the occurrence of maximal quantity for the 2 markers $90-120 \mathrm{mn}$ following the administration.

constant pour tous les animaux. Compte tenu de ce dernier aspect, de la faculté d'identification des P.P.E. par flottaison et de la vidange gastrique apparemment uniforme, quelle que soit la période du nycthémère, la mesure du T.T.M. gastro-iléal à l'aide de P.P.E. s'avère un procédé simple et relativement satisfaisant puisque les valeurs sont très proches de celles multiples et bien identifiées par la technique d'abattage (LAPLACE et al., 1975).

Le T.T.M. des P.P.E. est légèrement inférieur à celui de la P.S.P. pour le segment cæco-colique dans le cas d'élimination de crottes dures. On retrouve donc à nouveau pour les P.P.E. des valeurs proches de celles fournies dans les techniques d'abattage (Pickard \& Stevens, 1972). Un intérêt majeur de l'emploi des P.P.E. est la possibilité d'identifier les périodes de production de cæcotrophes puisque dans ce cas, le T.T.M. est ramené à environ 2 heures. Au demeurant, quel que soit le marqueur utilisé, il apparaît que les T.T.M. ne sont modifiés, en fonction de la période du nycthémère, qu'au niveau colique. Selon Gidenne et Poncet (1985), la plus forte densité de 
cæcotrophes dans l'estomac se situe entre 9 et $15 \mathrm{~h}$. Nous n'avons pas retrouvé une influence majeure de la présence ou non de cæcotrophes dans l'estomac sur la vidange gastrique de la P.S.P. et sur le transit bouche-iléon pour des essais effectués à $14 \mathrm{~h}$ et $20 \mathrm{~h}$.

Cette étude souligne la rapidité de transit bouche-iléon et l'importance du processus de séparation physique des phases solide et liquide dans le côlon proximal au cours de la période de production de fèces dures.

Compte tenu de cette dernière remarque, la mesure du T.T.M. chez le lapin à l'aide de P.P.E. s'avère un procédé simple et relativement satisfaisant.

Reçu en mai 1986.

Accepté en septembre 1986.

\section{Summary}

Value of indigestible particles to measure the gastrointestinal transit time in the rabbit

Measurement of the flow of digesta in the rabbit is complicated by the peculiar physiolegy and anatomy of the hindgut and the production of two types of faeces in this species. It might therefore require use of a two-marker technique for the liquid and solid phases, respectively.

In order to evaluate the changes in the flow of digesta, the mean transit time (M.T.T.) of indigestible polyethylene particles (P.E.P.) was compared to that of sulfonphenolphtalein (S.P.P.), for the gastro-ileal and caeco-colonic segments, respectively. The markers were given at different times during the day in order to estimate the possible differences due to production of soft and hard faeces, respectively.

In the stomach and small intestine, M.T.T. of P.E.P. exceeded by about 20 p. 100 that of S.P.P. During production of soft faeces, M.T.T. of both P.E.P. and S.P.P. were similar in the hindgut whereas with hard faeces P.E.P. was eliminated 20 p. 100 more rapidly than S.P.P.

A mean transit time of $90 \mathrm{mn}$ for P.E.P. was recorded in the gastro-ileal segment regardless of the period of the day. During elimination of soft faeces, M.T.T. of P.E.P. was less than $2 \mathrm{~h}$ and $4 \mathrm{~h}$ during that of hard faeces.

These results validate the use of P.E.P. alone as a tool for measuring the transit time and for identifying the defecation patterns in the rabbit.

Key words: Transit time, indigestible particles, caecotrophy.

\section{Références bibliographiques}

Barreiro M.A., McKenna R.D., Beck I.I., 1968. Determination of transit time in the human jejunum in the simple injection indicator-dilution technique. Am. J. Dig. Dis., 13, $222-232$.

Bechgand H., Christensen F.N., Davis S.S., Hardy J.G., Taylor M.J., Whalley D.R., WILSON C.G., 1985. Gastrointestinal transit of pellet systems in ileostomy subjects and the effect of density. J. Pharm. Pharmacol., 37, 718-721.

Chaussade S., Roche H., Khyari A., Couturier D., Guerre J., 1986. Mesure du temps de transit colique (T.T.C.) : description et validation d'une nouvelle technique. Gastroentérol. Clin. Biol., 10, 385-389. 
Cherbut C., Ruckebusch Y., 1984. Modifications de l'électromyogramme du côlon liées à l'ingestion de particules non digestibles chez le chien. Gastroenterol. Clin. Biol., 8, 955-959.

Cherbut C., Ruckebusch Y., 1985. The effect of indigestible particles on digestive transit time and colonic motility in dogs and pigs. Br. J. Nutr., 53, 549-557.

Gidenne T., Poncet C., 1985. Digestion, chez le lapin en croissance, d'une ration à taux élevé de constituants pariétaux : étude méthodologique pour le c. $^{1}$ cul de digestibilité apparente, par segment digestif. Ann. Zootech., 34, 429-446.

Laplace J.P., Lebas F., 1977. Le transit digestif chez le lapin. VII. Influence de la finesse du broyage des constituants d'un aliment granulé. Ann. Zootech., 26, 413-420.

Laplace J.P., Lebas F., Rioperez J., 1974. Le transit digestif chez le lapin. 1) Utilisation du Cérium-141 : Etude méthodologique et descriptive. Ann. Zootech., 23, 555-576.

Laplace J.P., Lebas F., Rioperez J., 1975. Le transit digestif chez le lapin. 2) Répartition de la radioactivité après ingestion d'aliment marqué au Cérium-141. Ann. Zootech., 24, 59-68.

Lawson M., 1986. Gut transit time measured by three markers. Proc. Nutr. Soc., 54 A.

Pairet M., Bouyssou T., Ruckebusch Y., 1986a. Colonic formation of soft faeces in rabbits : a role for endogenous prostaglandins. Am. J. Physiol., 250, G302-G308.

Pairet M., Bouyssou T., Auvergne A., Candau M., Ruckebusch Y., 1986b. Stimulation physico-chimique d'origine alimentaire et motricité digestive chez le lapin. Reprod. Nutr. Develop., 26, 85-95.

Pickard D.W., Stevens C., 1972. Digesta flow through the rabbit large intestine. Am. J. Physiol., 222, $1161-1166$.

Prud'hon M., Carles Y., Goussopoulos J., Koehl P.F., 1972. Enregistrement graphique des consommations d'aliments solide et liquide du lapin domestique nourri ad libitum. Ann. Zootech., 21, 347-353.

Snedecor G.W., Cochran W.G., 1967. Statistical Methods (6th Ed.). Ames, Iowa, Iowa State Univ. Press, 369-374.

Uden P., Colucci E.P., VAn Soest P.J., 1980. Investigation of chromium, cerium and cobalt as markers in digesta. Rate of passage studies. J. Sci. Fd. Agric., 31, 625-632. 